\title{
The effects of humming and pitch on craniofacial and craniocervical morphology measured using MRI
}

\author{
Abstract \\ Objectives/Hypothesis \\ Traditional voice research occurs within a phonetic context. Accordingly, pitch-related \\ contributions are inseparable from those due to articulator input. In humming, articulator \\ input is negligible. Using MRI we test the hypothesis that voice production is accompanied \\ by pitch-related adjustments unrelated to articulatory or postural input.
}

\section{Study Design and Method}

In this cross-sectional study, 10 healthy volunteers ( 5 males, 5 females, age 20-47, median 25 years) including singers (6 months-10 years tuition, median 2 years) and non-singers, were assessed to establish the lowest and highest notes they could comfortably sustain while humming over 20 seconds. With head position stable, mid-sagittal images were acquired while volunteers hummed these predetermined low and high notes. 22 craniocervical, angular and linear dimensions defined on these images were compared using One Way Repeated Measures ANOVA. Correlations between variables were sought using Pearson's correlation coefficient.

\section{Results}

We found significant differences between low and high note conditions in 6/22 measures and widespread pitch-related correlations between variables $(r \geq 0.66, P<0.05)$. Compared with low note humming, high note humming was accompanied by increased craniocervical angles opt/nsl and cvt/nsl ( $P=0.008$ and 0.002 respectively); widening of the C3-menton distance $(P=0.003)$, a rise of the larynx and hyoid in relation to the cranial base $(P=0.012$ and $<$ 0.001 respectively) and a decreased sternum-hyoid distance $(P=0.004)$.

\section{Conclusion}

Voice production is accompanied by pitch-related adjustments that are currently being masked by, or mistakenly attributed to, articulatory or postural input, identification of which could improve understanding of mechanisms underlying speech and song.

\section{Keywords}

Humming-pitch-craniofacial-craniocervical-morphology-MRI-vocal tract 


\section{Introduction}

Aristotle described voice as 'sound with a meaning' (1). In speech and song, meaning is conveyed to the listener by the symbolic use of sound (words) and by the tone of the voice, its pitch, strength and quality. According to the traditional source-filter theory of voice production, the fundamental frequency $(\mathrm{F} 0)$ of the voice and its harmonics are determined by the length and tension of the vocal folds $(2,3)$. As the sound wave passes through the vocal tract, it is shaped into the words we recognize by movement of the articulators, the lips, tongue, jaw and soft palate. The nature of the relationship between the pitch (or tone) of the voice and articulation is unclear $(4,5,6)$. We know that movements of the articulators are accompanied by compensatory adjustments of the pitch production mechanism $(7,8)$. Conversely, changes of voice pitch are accompanied by 'important modifications' in the position of the articulators during the production of a vowel sound $(5,9,10)$. A better understanding of the mechanisms underlying this apparent reciprocal relationship between pitch and articulation could contribute to explanations for a number of observations in voice literature that are not yet fully understood. For example, the presence of 'unintended compensatory adjustments' of the vocal tract during the rise and fall of the larynx (11), differences in the behaviour of the soft palate between speech and singing (12), and differences between vocal tract shapes for low and high pitched voices $(13,14)$.

Traditionally, voice research occurs within a phonetic context whereby speech or speechrelated sounds are used in experiments designed to investigate vocal adjustments during voice production (15-17). This means that when pitch is included as a variable, it is difficult to separate possible contributions arising solely from a change of pitch from those due to articulator movement. However, by humming we can produce sound over a wide pitch range but with negligible articulator input thus allowing movements of the vocal tract and related structures to be investigated within a non-phonetic context. Previously, we successfully examined the vocal tract and related structures within the context of their direct and indirect structural attachments to the cranium, cervical spine and sternum by combining the superior soft tissue definition of magnetic resonance imaging (MRI) with bony reference points used in cephalometry (Rest paper ref). Our results highlighted the need to consider the vocal tract and related structures within the context of their wider structural relationships if important information is not to be missed. In this experiment, we used the same method to examine voice production within a non-phonetic context by investigating vocal changes in subjects asked to hum at low and high pitches. The aims of this study are twofold: to investigate the 
hypothesis that voice production is accompanied by pitch-related adjustments that are independent of those arising from articulator or jaw-related input and that are currently being masked by, or mistakenly attributed to, articulatory or postural changes; and to look for correlations between vocal structures when subjects hum low and high notes.

\section{Methods}

\section{Recruitment}

Twelve healthy volunteers were recruited to the study. All but one (who had a tonsillectomy and adenoidectomy as a child) had no history of speech or hearing pathology. As subjects were required to be able to voice and sustain notes of different pitches over 20 seconds while lying in an enclosed space, exclusion criteria included a history of claustrophobia and an inability to maintain a closed mouth position within this 20 second time frame, in addition to the presence of contraindications to MRI such as pacemakers and metallic orthodontic appliances. Approval from Grampian Research Ethic Committee (now North of Scotland Research Ethics Service) was obtained and all subjects gave written informed consent.

\section{Procedure}

Before the experiments, NM met with the volunteers to explain the procedure and to establish the lowest and highest notes that could be comfortably, steadily and confidently hummed while breathing out over 20 seconds: volunteers were instructed to look straight ahead without flexing or extending their head, to keep their lips and teeth together, and to rest the tongue comfortably against the roof of the mouth. Beginning with humming a middle range note played on a piano tuned to concert pitch, volunteers ascended (and descended) the scale until a point was found where it was no longer comfortable and the note was beginning to sound strained. By retracing one or two steps, the lowest and highest notes that could be comfortably hummed were obtained. A recording was produced containing the notes for all volunteers. For the whole group these ranged from $98 \mathrm{~Hz}(\mathrm{G} 2)$ to $1047 \mathrm{~Hz}$ (C6) where C4 is middle $\mathrm{C}$.

During acquisition of MRI data, volunteers lay supine and were required to adopt a relaxed posture in the MRI scanner. They were instructed to look straight head while holding the lips and teeth together, to rest the tongue comfortably against the hard palate and to maintain this position during production of low and high notes. Individuals were imaged with the head placed in a Sense-Neurovascular array-16 element coil. Deformable foam wedges were used 
to make the subject comfortable and to restrain the head position. Ear plugs and headphones helped attenuate the scanner noise and the headphones enabled presentation of the previously determined low and high notes. Para-sagittal images were obtained with a 3.0 T Achieva MR system (Philips, Best, Holland) using a turbo spin echo pulse sequence with the following parameters: field of view (FOV) $340 \mathrm{~mm}$ x $340 \mathrm{~mm}$; a 768 by 768 matrix; repetition time $4106 \mathrm{~ms}$; echo time $100 \mathrm{~ms}$; 6 slices $4.0 \mathrm{~mm}$ thick with a gap of $1.0 \mathrm{~mm}$ centred on the midsagittal plane. The number of slices was dictated by the need to optimize image resolution within the time constraint of a single breath (about 20 seconds). The FOV extended from just above the pituitary fossa to the sternal notch, taking in the width of the whole head and neck.

Each individual was scanned three times while adopting the above posture: at rest during quiet breathing, while performing first a low note hum (LNH) and then a high note hum (HNH). To enable each individual to achieve the predetermined low and high notes, the note was played through the headphones and the subject asked to breathe in and to start humming the note while breathing out over twenty seconds. As they began to hum, the scanning sequence started. The MRI slice closest to the mid-sagittal plane was chosen for analysis (identified by the presence of the pituitary fossa, the tip of the odontoid process, the spinal processes and the outline of the trachea and spinal cord).

\section{Image analysis}

Images were converted from DICOM to Bitmap format using ImageJ (18). Software tools, developed by the University of Manchester, UK (19), were used manually to mark reference points as shown in Figure 1 and described in Table 1. From these points, a program was written to automatically measure the 22 variables chosen to describe the craniocervical, angular, craniocaudal and antero-posterior dimensions illustrated in Figure 1 and listed in Table 2.

\section{Statistical analysis}

Statistical analysis was performed using Sigmastat (v11, Systat Software, Inc.). All data were normally distributed with equal variance. One Way Repeated Measures ANOVA was used to investigate differences between groups and post-hoc ANOVA group comparisons were performed using the Holm-Sidak test with significance set at $P \leq 0.05$. Correlations between all 22 variables were sought using Pearson's product-moment correlation coefficient. To avoid the charge that observed correlations could be attributed to those present in the Rest 
condition, Rest data were subtracted from $\mathrm{LNH}$ and $\mathrm{HNH}$ data. Correlations were then sought between variables using data solely attributable to the change of state from Rest to $\mathrm{LNH}$, and from Rest to HNH. For all tests $P<0.05$ was taken to indicate statistical significance.

\section{Results}

Ten out of twelve subjects met the criteria for inclusion into the study. One subject was unable to adopt the necessary tongue position with the tongue dorsum resting comfortably against the hard palate. Another adopted a hyper-extended neck position and was unable to produce voice comfortably without undue strain. We obtained a full data set for the remaining ten subjects ( 5 males, 5 females; age range 20-47 with a median of 25 years). Subjects included singers (one a professional singer) with singing tuition ranging from 6 months to 10 years (median 2 years), and non-singers. The dimensions of variables shown in Figure 1 are described in Table 3. Results for the Rest condition have been reported previously (Rest Paper ref.). In this paper, we focus upon significant differences between Rest and the low note hum (LNH) and high note hum (HNH) conditions.

\section{Changes in morphology with pitch}

We observed significant differences between Rest, LNH and HNH for 9 of the 22 variables considered. Post-hoc analysis revealed significant differences for 6 out of 22 variables between $\mathrm{LNH}$ and $\mathrm{HNH}, 4$ out of 22 variables between Rest and LNH, and 5 out of 22 variables between Rest and HNH. These results are illustrated in Figure 2 and further described below.

Figure 2.1 shows that variables found to be significantly different between LNH and HNH fall into three broad groups; changes involving the upper craniocervical angles, those involving craniocaudal variables, and one antero-posterior variable. On average, the switch from $\mathrm{LNH}$ to $\mathrm{HNH}$ was associated with increased craniocervical angles opt/nsl and cvt/nsl ( $P$ $=0.008$ and 0.002 respectively), shorter perpendicular distances from the base of the cranium to the larynx (1-nsl) and the hyoid (hy-nsl) $(P=0.012$ and $P<0.001$ respectively) and greater distances between the sternum and the hyoid (stern-hy) and between $\mathrm{c} 3$ and the menton (c3me) $(P<0.001$ and 0.003 respectively). Moving from Rest to LNH was also associated with an increased lower craniocervical angle evt/nsl $(P=0.018)$, but now there was an increased distance between the hyoid and the cranial base $(\mathrm{P}<0.001)$, a shorter sternum-hyoid distance $(P<0.001)$, and an increased distance of the epiglottis tip from the posterior pharyngeal wall 
(et-ppw) $(P=0.041)$ (Figure 2.2). Moving from Rest to HNH was associated with increased upper (opt/nsl, cvt/nsl) and lower (evt/nsl) craniocervical angles $(P=0.008,0.002$ and 0.018 respectively), and greater distances between $\mathrm{c} 3$ and both the hyoid (hy-c3) $(P=0.003)$ and the menton $(P=0.003)$ (Figure 2.3).

\section{Correlation analysis}

Widespread correlations were observed between craniocervical, angular and linear variables. These correlations were different, however, depending upon whether the subjects were humming a low (Table 4) or a high (Table 5) note, and both were different from those previously published at rest (in this journal-as accompanying paper). For clarity, the term 'height' will be used here to refer to the perpendicular distances of the larynx, hyoid, uvula (tip) and epiglottis (tip) from the cranial base.

In Tables 4 and 5, uncorrelated variables are omitted (in LNH, the distances between the larynx and hyoid (l-hy) and between the uvula tip and the posterior pharyngeal wall (ut-ppw), and in $\mathrm{HNH}$, the height of the hyoid and the angle of the epiglottis (ae)). The remaining variables are arranged, as far as possible, to highlight relationships between them (for all variables, $r \geq 0.63$ ). Since this is a cross-sectional study, it is not possible to directly compare correlations between $\mathrm{LNH}$ and $\mathrm{HNH}$ for any one individual therefore reference can only be made to changes across the group.

Correlations observed in low note humming

Table 4 shows two broad patterns of correlations associated with humming a low note; groups of intercorrelated variables (with overlapping of groups 1-3 and 4-5 listed below), and correlations independently associated with each variable (with the exception of the distances separating the hyoid from both the menton (hy-me) and from the mandible). The groups of intercorrelated variables (from left to right) are comprised as follows:

1. the craniocervical angles opt/nsl and cvt/nsl with hyoid height (hy-nsl) ( $r \geq 0.67)$;

2. the craniocervical angle cvt/nsl with the heights of the hyoid, larynx (l-nsl), and epiglottis (et-nsl) $(r \geq 0.64)$;

3. the heights of the uvula (ut-nsl) and epiglottis (et-nsl) with the angle of the soft palate (asp) $(r \geq 0.66)$; 
4. the length of the soft palate (pns-ut) with the sternum-hyoid distance (stern-hy) and the width of the laryngeal tube opening (ltw) $(r=0.69)$; and,

5. the width of the laryngeal tube opening with the distance of the hyoid from c3 (hyc3), the menton (hy-me) and the mandible (hy-ma) $(r \geq 0.8)$.

Correlations observed in high note humming

Examination of Table 5 shows one group of intercorrelated variables, between the hyocervical distance and the distances of the hyoid from both the menton and mandible $(r \geq$ 0.79), and 9 partially overlapping pairs of correlated variables. From left to right these are between:

1. the craniocervical angles opt/nsl and cvt/nsl $(r=0.82)$;

2. the thyro-hyoid distance (l-hy) and larynx height (l-nsl) $(r=0.67)$;

3. larynx height and epiglottis height (et-nsl) $(r=0.88)$;

4. uvula height (ut-nsl) and the angle of the soft palate (asp) $(r=0.77)$;

5. the angle of the soft palate and the width of the laryngeal tube opening (ltw) $(r=$ $0.63)$;

6. the width of the laryngeal tube opening and the distance of the uvula tip from the posterior pharyngeal wall (ut-ppw) $(r=0.76)$;

7. the distance of the uvula tip from the posterior pharyngeal wall and the minimal distance separating the uvula from the posterior pharyngeal wall (u-ppw) ( $r=$ $0.76)$

8. the oropharyngeal airway at the uvula tip (pt-ppw-ut) and epiglottis tip (pt-ppw-et) ( $r$ $=0.78) ;$ and,

9. the oropharyngeal airway at the epiglottis tip and the distance between the epiglottis tip and the posterior pharyngeal wall (et-ppw) $(r=0.65)$.

In addition to these groups, Table 5 shows each variable to be independently associated with other variables with the exception of the following 8 variables: thyro-hyoid distance, epiglottis height, the angle of the soft palate, the width of the laryngeal tube opening, the 
distance of the uvula tip from the posterior pharyngeal wall, the minimal distance separating the uvula from the posterior pharyngeal wall and the oropharyngeal airway at the tips of the uvula and epiglottis.

Correlations common to both low note humming and high note humming

Correlations common to both low and high note humming conditions are strongly and very strongly correlated $(r \geq 0.77)$ with the exception of 3 pairs of variables (the craniocervical angle opt/nsl and the $\mathrm{c} 3$-menton distance in $\mathrm{LNH}$, the oropharyngeal airway at the epiglottis tip and the distance between the epiglottis tip and the posterior pharyngeal wall in $\mathrm{HNH}$, and the craniocervical angle cvt/nsl and uvula height in LNH and HNH) (Table 6). From left to right, correlated variables common to $\mathrm{LNH}$ and $\mathrm{HNH}$ are as follows:

1. the craniocervical angles opt/nsl and cvt/nsl with the c3-menton distance;

2. the craniocervical angle cvt/nsl with uvula height (ut-nsl);

3. larynx height (l-nsl) and epiglottis height (et-nsl);

4. uvula height with the craniocervical angle cvt/nsl and the angle of the soft palate (asp);

5. the angle of the soft palate with uvula height;

6. the hyocervical distance (hy-c3) with the distance of the hyoid from both the menton (hy-me) and the mandible (hy-ma);

7. the width of the airway at the tips of the uvula (pt-ppw-ut) and epiglottis (pt-ppw-et); and,

8. the width of the airway at the epiglottis tip and the distance separating the epiglottis from the posterior pharyngeal wall (et-ppw).

Differences between correlations observed for LNH and HNH

A study of Tables 4, 5 and 6 shows a number of differences between LNH and HNH. The most striking findings are the absence of associations with hyoid height (hy-nsl) and the length of the soft palate (pns-ut) in $\mathrm{HNH}$, and the different patterns of correlations in $\mathrm{LNH}$ and $\mathrm{HNH}$ found in association with the hyocervical distance (hy-c3). Correlations associated 
with hyoid height and the length of the soft palate in LNH, and those associated with the hyocervical distance in LNH and $\mathrm{HNH}$ are illustrated in Figure 3.

\section{Discussion}

In this study, we showed the switch from humming a low note to humming a high note to be accompanied by significant changes affecting not only the vocal tract and related structures but also extending to other regions of the head and neck. Additionally, changing from one state to another (Rest, LNH or HNH) resulted in different patterns of correlated activity. These results demonstrate the presence of coordinated, pitch-dependent structural adjustments during voice production under conditions where articulator input is negligible, thus supporting our hypothesis that voice production at different pitches is accompanied by pitch-related adjustments that are independent of those secondary to articulatory or postural input.

On average, the change from humming a low note to humming a high note was accompanied by an increase in the upper craniocervical angles, a rise of the hyoid and larynx in relation to the cranial base, and greater distances between the sternum and hyoid, and between $\mathrm{C} 3$ and the menton. Finding a rise in pitch to be associated with greater craniocervical angles was unexpected and raised the possibility that subjects may have unintentionally flexed or extended their heads during image acquisition. To investigate this, we examined unregistered images obtained from volunteer 3 who possessed the widest pitch range of all volunteers (147 Hz-1047 Hz). Figure 4 shows tracings obtained during low and high note humming and superimposed without translation or rotation. This shows negligible outward postural changes in the move from humming a low note to humming a high note. The local change in the posterior neck outline overlying C3 is the only outward indicator of more widespread adjustments involving the cervical (and thoracic) spine, supralaryngeal airway, vocal structures (soft palate, tongue, larynx, hyoid and epiglottis) and trachea. Hence, this not only illustrates the widespread pitch-dependent adjustments but also shows that our demonstration of significant pitch-related changes affecting the craniocervical angles is valid and not merely the result of unintentional positional changes during image acquisition.

Craniocervical changes have been reported in association with voice production but these studies were based within a phonetic context and changes attributed to jaw opening (20) or the development of a more forward head posture (21). In a little known study, Mitchinson 
and Yoffey (1948) (22) used X-ray to investigate vocal fold changes while humming at each end of the vocal range. However, in addition to reporting pitch-related changes of the vocal folds, the authors also observed that the change from humming a low note to humming a high note was accompanied by increased antero-posterior thickness of the pre-vertebral soft tissues. Although there were no other observations of pitch-related cervical changes, the following findings were reported: lengthening of the vestibular folds; upward movement of the larynx and, to a lesser extent, the hyoid; approximation of the larynx to the hyoid; rotation, upward and forward movement of the hyoid and epiglottis; narrowing of the laryngeal tube; shortening and widening of the supraglottic space; widening of the anteroposterior supraglottic cavity (thought to be secondary to forward hyoid movement) and straightening of the anterior border of the trachea. With the exception of vestibular fold length, which was not measured in this study, examination of Table 3 and Figure 4, and correlations observed in association with the laryngeal tube width (indicating hyoid rotation) in LNH (Table 4 and Figure 3.3), shows that our results are in full agreement with these findings. However, by examining vocal structures within the wider context of their direct and indirect structural attachments to the cranium, cervical spine and sternum we are also able to demonstrate greater involvement of craniocervical structures during the production of voice of different pitches than has hitherto been suspected, changes which could account for the increased antero-posterior thickness of pre-vertebral tissues commented upon by Mitchinson and Yoffey and evident in Figure 4.

As far as we are able to establish, this is the first study to report pitch-related changes involving the craniocervical angles in a non-phonetic context, a view supported by reports of pitch-related involvement of the neck muscles during phonation $(23,24)$. An explanation for this finding may lie in recognising the common nerve root origin of the nerves supplying postural neck and hyoid muscles (25). These findings are important and require further investigation as the implications are wide-ranging. For example, a better understanding of structural adjustments accompanying changes of pitch might help explain cervical curvature changes while singing, the increased incidence of cervical abnormalities in professional opera singers compared with non-singers $(20,21,26,27)$, and the development of voice difficulties in some patients (especially female singers) following cervical spine surgery, (28). These findings are also relevant in the light of recent suggestions that cervical muscles might have a role in 'fine-tuning' during pitch production (27). To date, other than the knowledge of a 
close association between pitch and posture, a coherent mechanism that fully accounts for cervical involvement has yet to be presented.

The requirement for rapid, precise and finely coordinated movement of vocal muscles during the production of speech and song is long recognized $(29,30)$. It is also acknowledged that the shape, size and position of vocal structures depend upon the balance of muscular tensions throughout the vocal system (31). However, details of the mechanisms underlying this highly complex and coordinated activity remain unclear. Although reports and suggestions of synergistic activity between vocal structures are increasing in voice research literature, $(12,20,32-36,36-38)$ a coherent framework within which to explain these observations is so far lacking $(39,40)$. We suggest that knowledge and awareness of pitch-dependent patterns of correlations could provide the basis for such a framework within which it may be possible to further our understanding of mechanisms underlying existing literature observations, and from which it may be possible to develop testable hypotheses concerning a wide range of speech and tonal-related phenomena for subsequent investigation with, for example, MRI and electromyography (EMG).

The power of this approach is demonstrated by its ability to cast light upon mechanisms underlying observations of, for example, the 'unintended compensatory adjustments' of the vocal tract (ref?) that accompany the rise and fall of the larynx; differences in soft palate behaviour between speech and singing; and differences in the shape and size of the vocal tract for low and high notes. Figure 3.2 shows soft palate length to be positively correlated with the height of the larynx. Hence, lowering of the larynx is associated with a longer soft palate and a rise of the larynx with a shorter soft palate. However, the same Figure also shows that associations between the length of the soft palate and larynx height are not to be considered in isolation. The presence of non-topographical correlations (where variables have no reference points or lines in common) as, for example, between soft palate length and both the width of the laryngeal tube opening and the sternum-hyoid distance, point to the existence of underlying coordinating mechanisms (41). This suggests that the relationship between soft palate length and larynx height is only one part of a particular pattern of possible adjustments that affect the size and shape of the vocal tract. Since the angle and height of the soft palate can change in addition to its length, pitch-related correlations independently associated with these variables (Tables 4 and 5) also need to be taken into account when considering changes in soft palate behaviour between speech and singing. 
The number of variables and the different combinations of their possible interactions (Tables 4 and 5) indicate that the size and shape of the vocal tract can be altered in different ways. However, the patterns of highly and very highly correlated variables $(r \geq 0.79)$ associated with the hyocervical distance, hy-c3, warrant special attention (Figs.3.4 and 3.5). The hyocervical distance is a measure of the width of the airway. In LNH, hyocervical distance is positively associated with both the velopharyngeal opening (the minimal distance separating the uvula from the posterior pharyngeal wall, u-ppw) and the sagittal width of the laryngeal tube opening, and negatively associated with the distances from the hyoid to the menton and the mandible. In $\mathrm{HNH}$, the hyocervical distance is negatively associated with the height of the uvula tip (ut-nsl) and the distances of the hyoid from both the menton and the mandible. Like the variables associated with soft palate length, non-topographically associated variables between hy-c3 and u-ppw in LNH, and hy-c3 and ut-nsl in $\mathrm{HNH}$ indicate the presence of underlying coordinating mechanisms that have a material influence upon the size and shape of the vocal tract and, in particular, of two regions recognised for their importance in the singing voice; the velopharyngeal and laryngeal openings.

Only one of the volunteers in this study was a professional (opera) singer. It is well-known that trained singers adopt singing techniques that encourage a low larynx position $(43,44)$. In this study, this individual showed opposite results from those for the remainder of the group for all six variables found to be significantly different between low and high note humming. Hence, in the professional singer, the switch from humming a low note to humming a high note was accompanied by smaller craniocervical angles, greater distances of the hyoid and larynx from the cranial base, and shorter distances between the sternum and hyoid, and between $\mathrm{c} 3$ and the menton. The significance of these findings is currently the subject of investigation.

\section{Implications}

The findings of this study do not sit well with the traditional (linear) Source-Filter theory of voice production. The presence of significant pitch-related adjustments and of widespread patterns of pitch-dependent correlations under conditions where articulatory and postural input is negligible suggests that pitch-related vocal fold changes do not occur in isolation but, instead, occur as part of more widespread pitch-related adjustments throughout the head, neck and even, as indicated by thoracic spine involvement (Fig. 4) the body. "Mutual 
interdependence" between the source of the voice, the larynx, and the vocal tract is an old idea (e.g., Lennox Browne, 1875) (45). However, given increasing recognition of the importance of non-linear phenomena in voice production $(46,47)$, the lack of a full understanding of their underlying mechanisms $(48,49)$, a long-held view of the requirement to look beyond "the parts" if we are to understand the function of "the whole (vocal) system" and acknowledgement that current theories do not adequately account for a wide range of speech, language and tonal-related phenomena $(50,51)$, we suggest that this is a view that warrants serious re-examination. Overall, the results of this study lead us to put forward a bold and thought-provoking hypothesis that the ability to speak or to sing is superimposed upon a robust underlying pitch-adjusting system where perturbation by, for example, the rapid articulatory movements necessary for the production of speech, results in on-line, widespread and reflexive changes which serve to maintain a steady acoustic output.

\section{Limitations}

The small number of subjects is an important limitation of this study. Additionally, mixed sexes, varying ages, heights, weights, pitch ranges, voice classifications, singing experience/training and vocal strategies, all factors known to influence vocal dimensions, mean that great care is needed in the interpretation of these results (52). More work is necessary to confirm the validity of these findings. Nevertheless, the implication that pitch and articulation need to be separately accounted for in voice production experiments is important and warrants consideration. Future studies would be improved by using clearly defined populations such as healthy male tenors since trained singers are more likely than non-singers to be confident in the use and control of their voice. Another important limitation of this study is the supine position required by the MR scanner. This could be overcome by using a positional MRI scanner, designed for use in the upright position, which would allow investigation of voice production under more realistic conditions.

\section{Conclusion}

Traditional voice research largely focuses on the effect of speech-related activity upon the vocal tract, larynx and articulators. In this study we used MRI to explore the hypothesis that voice production is accompanied by pitch-related adjustments that are independent of those secondary to articulator or jaw-related input and that may be masked by, or mistakenly attributed to, articulatory or postural changes. By reducing articulatory and postural input to a 
minimum, we found significant extralaryngeal adjustments to accompany the switch from humming a low note to humming a high note and uncovered widespread patterns of pitchdependent correlations between variables. Previously unreported findings of pitch-related changes of the upper craniocervical angles demonstrate the importance of considering vocal structures and the airway within the context of their wider structural relationships if important information is not to be missed. The discovery of widespread pitch-dependent adjustments and associations provides a framework for the design of testable hypotheses which could further understanding of coordinated mechanisms underlying speech and song and shed new light upon a wide range of speech, language and tonal phenomena.

\section{References}

(1) Hutchins R. The Works of Aristotle: Volume I. ; 1952. p. 652.

(2) Müller J. Elements of Physiology. translation) W.Taylor and J.Walton 1840:1011.

(3) Fant G. Acoustic Theory of Speech Production. : Mouton De Gruyter; 1970.

(4) Bell-Berti F, Krakow R, Gelfer C, Boyce S. Anticipatory and carryover effects: Implications for models of speech production. Producing speech: Contemporary issues for Katherine Safford Harris 1995:77-98.

(5) $\mathrm{Hu}, \mathrm{F}$. Tonal effect on vowel articulation in a tone language. International Symposium on Tonal Aspects of Languages: With Emphasis on Tone Languages: ISCA; 2004.

(6) Zatorre RJ, Gandour JT. Neural specializations for speech and pitch: moving beyond the dichotomies. Philosophical Transactions of the Royal Society B: Biological Sciences 2008;363(1493):1087.

(7) Sawashima M, Hirose H, Yoshioka H, Kiritani S. Interaction between articulatory movements and vocal pitch control in Japanese word accent. Phonetica 1982;39(4-5):188198.

(8) Vilkman E, Sonninen A, Hurme P, Körkkö P. External laryngeal frame function in voice production revisited: A review. Journal of Voice 1996;10(1):78-92.

(9) Parmenter CE, Trevino SN, Bevans CA. The Influence of a Change in Pitch on the Articulation of a Vowel. Language 1933;9(1):72-81.

(10) Curry R. The mechanism of pitch change in the voice. J.Physiol.(Lond.) 1937;91(3):254.

(11) Sulter AM, Miller DG, Wolf RF, Schutte HK, Wit HP, Mooyaart EL. On the relation between the dimensions and resonance characteristics of the vocal tract: a study with MRI. Magn.Reson.Imaging 1992;10(3):365-373. 
(12) Fowler LP, Morris RJ. Comparison of nasalance between trained singers and non-singers [electronic resource]. 2004.

(13) Narayanan S, Bresch E, Tobin S, Byrd D, Nayak K, Nielsen J. Resonance tuning in soprano singing and vocal tract shaping: Comparison of sung and spoken vowels. J.Acoust.Soc.Am. 2006;119:3305.

(14) Takemoto H, Kitamura T, Honda K, Masaki S. Deformation of the hypopharyngeal cavities due to $\mathrm{F} 0$ changes and its acoustic effects. Acoustical Science and Technology 2008;29(4):300-303.

(15) Ventura SR. Application of MRI and biomedical engineering in speech production study. Comput.Methods Biomech.Biomed.Engin. 2009;12(6):671-681.

(16) Tokuda IT, Zemke M, Kob M, Herzel H. Biomechanical modeling of register transitions and the role of vocal tract resonators. J.Acoust.Soc.Am. 2010;127:1528.

(17) Munhall K. Functional imaging during speech production. Acta Psychol. 2001;107(95):117.

(18) Rasband W.S., ImageJ, U.S. National Institutes of Health, Bethesda, Maryland, USA, http://rsb.info.nih.gov/ij/, 1997-2009.

(19) Available at:

http://personalpages.manchester.ac.uk/staff/timothy.f.cootes/software/am_tools_doc/index.ht $\underline{\mathrm{ml}}$.

(20) Scotto Di Carlo N. Cervical column abnormalities in professional opera singers. Folia Phoniatr.Logop. 1998;50:212-218.

(21) Johnson G, Skinner M. The demands of professional opera singing on cranio-cervical posture. Eur.Spine J. 2009 Apr;18(4):562-569.

(22) Mitchinson AGH, Yoffey JM. Changes in the vocal folds in humming low and high notes. A radiographic study. J.Anat. 1948;82(Pt 1-2):88.

(23) Yamawaki Y. Forward movement of posterior pharyngeal wall on phonation. Am.J.Otolaryngol. 2003 Nov-Dec;24(6):400-404.

(24) Pettersen V, Westgaard RH. The activity patterns of neck muscles in professional classical singing. Journal of Voice 2005;19(2):238-251.

(25) Standring S. Gray's Anatomy. The Anatomical Basis of Clinical Practice. Churchill Livingstone, Edinburgh 2005:555.

(26) Sonninen A, Hurme P, Laukkanen AM. The external frame function in the control of pitch, register, and singing mode: radiographic observations of a female singer. J.Voice 1999 Sep;13(3):319-340. 
(27) Honda K, Hirai H, Masaki S, Shimada Y. Role of vertical larynx movement and cervical lordosis in F0 control. Lang.Speech 1999;42(4):401.

(28) Yue WM, Brodner W, Highland TR. Persistent swallowing and voice problems after anterior cervical discectomy and fusion with allograft and plating: a 5-to 11-year follow-up study. European Spine Journal 2005;14(7):677-682.

(29) Kent RD. The uniqueness of speech among motor systems. Clinical Linguistics \& Phonetics 2004;18(6):495-505.

(30) Honda K, Maeda S, Sugito M. Articulation changes in different voicing patterns. 2007.

(31) Kuehn DP, Folkins JW, Cutting CB. Relationships between muscle activity and velar position. Cleft Palate J. 1982 Jan;19(1):25-35.

(32) Fritzell B. The velopharyngeal muscles in speech. An electromyographic and cineradiographic study. Acta Otolaryngol. 1969;:Suppl 250:15.

(33) Birch P, Gumoes B, Stavad H, Prytz S, Bjorkner E, Sundberg J. Velum behavior in professional classic operatic singing. J.Voice 2002 Mar;16(1):61-71.

(34) Ysunza A, Vazquez MC. Velopharyngeal sphincter physiology in deaf individuals. The Cleft Palate-Craniofacial Journal 1993;30(2):141-143.

(35) Amelot A, Crevier-Buchman L, Maeda S. Observations of the velopharyngeal closure mechanism in horizontal and lateral directions from fiberscopic data. Proc.of the 15th $\mathrm{ICPhS}$ 2003:3021-3024.

(36) Honda, H. Physiological factors causing tonal characteristics of speech: from global to local prosody. Speech Prosody 2004, International Conference: ISCA; 2004.

(37) Erickson D, Iwata R, Endo M, Fujino A. Effect of tone height on jaw and tongue articulation in Mandarin Chinese. Proc.Tonal aspects of languages, Beijing 2004.

(38) Kokawa T, Saigusa H, Aino I, Matsuoka C, Nakamura T, Tanuma K, et al. Physiological studies of retrusive movements of the human tongue. J.Voice 2006 Sep;20(3):414-422.

(39) Kent RD. Research on speech motor control and its disorders A review and prospective. J.Commun.Disord. 2000;33(5):391-428.

(40) Perkell J, Matthies M, Lane H, Guenther F, Wilhelms-Tricarico R, Wozniak J, et al. Speech motor control: Acoustic goals, saturation effects, auditory feedback and internal models. Speech Commun. 1997;22(2-3):227-250.

(41) Solow B, Tallgren A. Head posture and craniofacial morphology. Am.J.Phys.Anthropol. 1976 May;44(3):417-435.

(42) Ohala J, Hirose H. The function of the sterno-hyoid muscle in speech. Annual Report 1969;4:41-44. 
(43) Iwarsson J, Sundberg J. Effects of lung volume on vertical larynx position during phonation. Journal of Voice 1998;12(2):159-165.

(44) Neuschaefer-Rube C, Wein B, Angerstein W, Klajman S. MRI examination of laryngeal height during vowel singing. Folia Phoniatr.Logop. 1996;48(4):201-209.

(45) Browne L. On Medical Science in Relation to the Voice as a Musical Instrument. Proceedings of the Musical Association 1875;2:94-110.

(46) Titze IR, Story BH. Acoustic interactions of the voice source with the lower vocal tract. J.Acoust.Soc.Am. 1997;101:2234.

(47) Titze IR. Nonlinear source-filter coupling in phonation: Theory. J.Acoust.Soc.Am. 2008;123:2733.

(48) The effect of the hypopharyngeal and supra-glottic shapes on the singing voice. Proceedings of the Stockholm Music Acoustics Conference (SMAC 2003), II; 2003.

(49) Using imaging and modeling techniques to understand the relation between vocal tract shape to acoustic characteristics. Proceed. of the Stockholm Music Acoustics Conference; 2003.

(50) Oudeyer PY. The Self-Organization of Speech Sounds. Arxiv preprint cs.LG/0502086 2005.

(51) Xu Y. Principles of Tone Research.

(52) Roers F, Mürbe D, Sundberg J. Voice classification and vocal tract of singers: A study of x-ray images and morphology. J.Acoust.Soc.Am. 2009;125:503.

Are there names and page numbers associated with 48,49 ? 


\section{Tables and Figures LNH v HNH Paper Draft 3}

Figure 1 Bony and soft tissue landmarks chosen for craniocervical, angular and linear variables

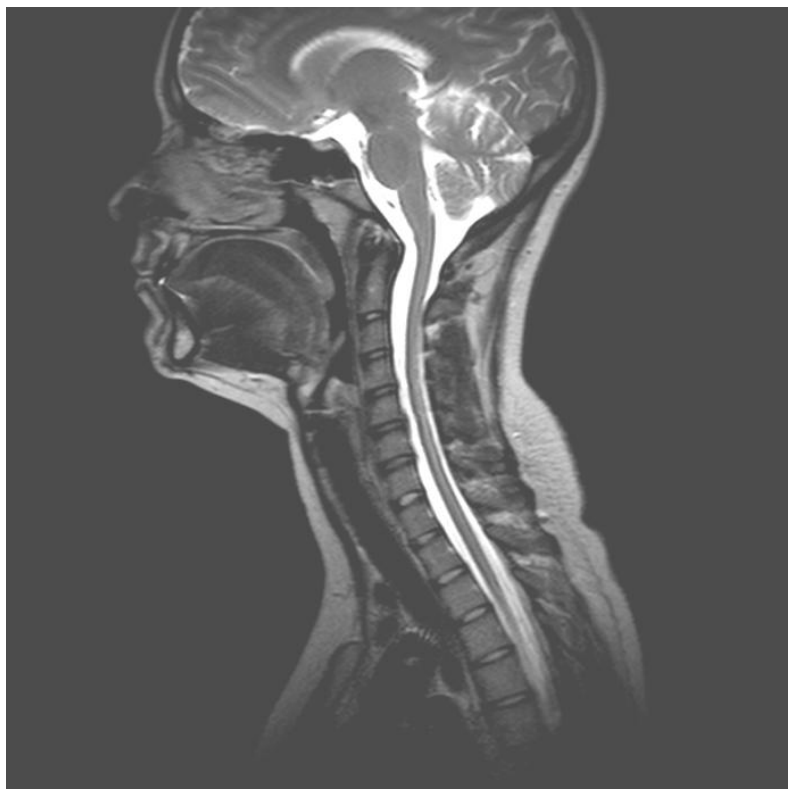

Mid-sagittal MR image

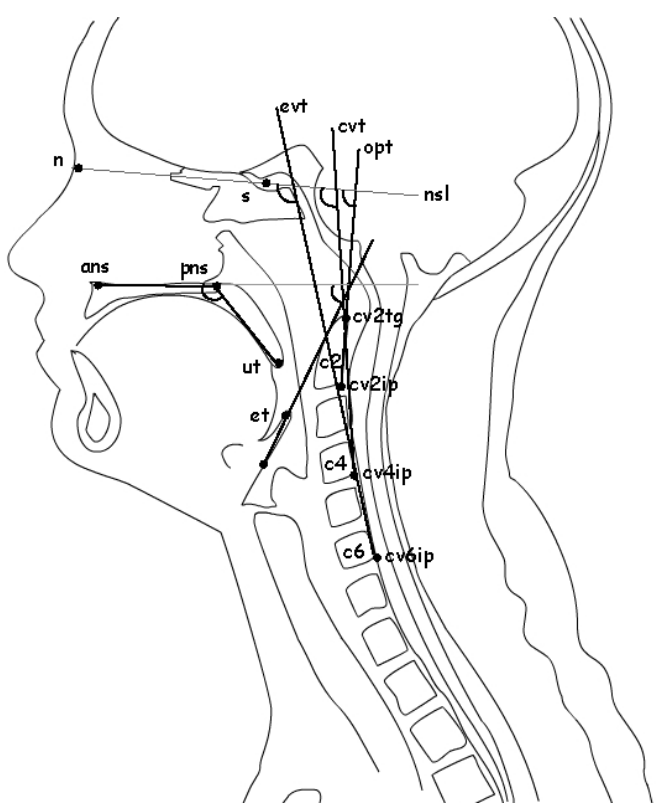

Craniocervical and angular variables

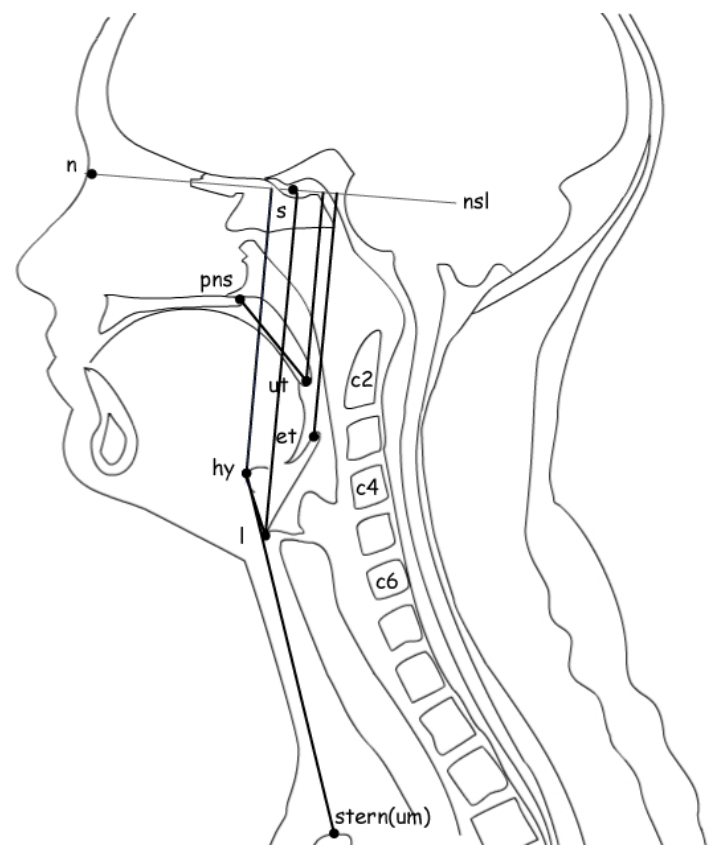

Craniocaudal variables

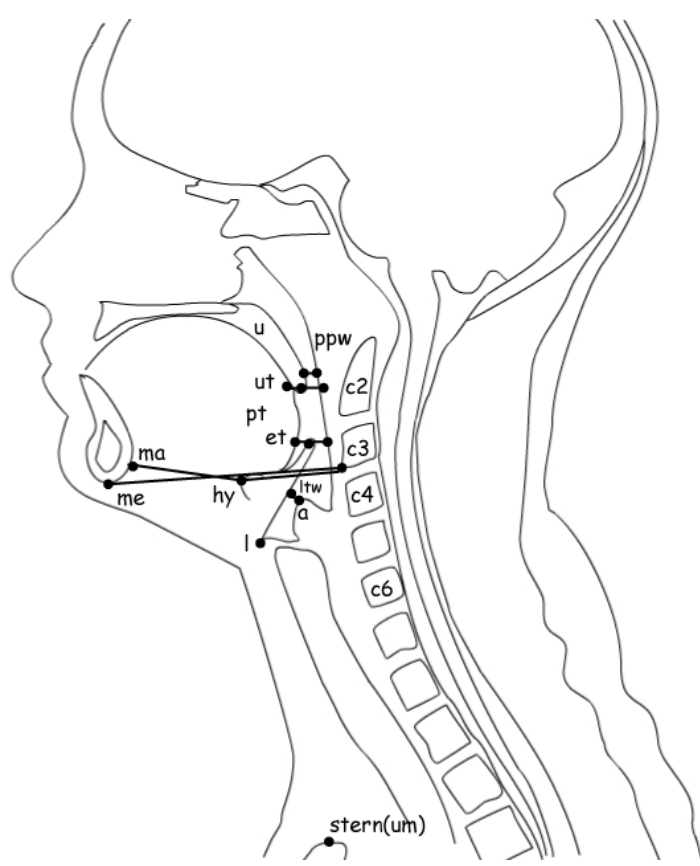

Antero-posterior variables 
Table 1 Bony and soft tissue landmarks, abbreviations and definitions

\begin{tabular}{|c|c|}
\hline Reference Point & Definition \\
\hline $\mathbf{a}$ & Superior margin of arytenoid cartilage \\
\hline ans & The most anterior point of maxilla at level of hard palate \\
\hline asp & Angle of soft palate \\
\hline et & Epiglottis tip \\
\hline cv & Cervical vertebra \\
\hline cv2tg & The point at the superior extremity of the odontoid process of cv2 \\
\hline cv2ip & The most infero-posterior point on the body of cv2 \\
\hline cv4ip & The most infero-posterior point on the body of cv4 \\
\hline cv6ip & The most infero-posterior point on the body of cv6 \\
\hline c3 & The most antero-inferior point on the body of cv3 \\
\hline cvt & A line through cv2tg and cv4ip \\
\hline evt & A line through cv4ip and cv6ip \\
\hline hy & The antero-superior margin of outer cortex of hyoid bone \\
\hline $\mathbf{l}$ & The anterior point of vocal folds \\
\hline Itw & Laryngeal tube width \\
\hline ma & The outer margin of posterior cortex of mandible \\
\hline me & Menton-the most inferior point of bony chin \\
\hline $\mathbf{n}$ & A point overlying the nasion \\
\hline nsl & A line joining ' $n$ ' and 's' reflecting orientation of anterior cranial base \\
\hline opt & A line through cv2tg and cv2ip \\
\hline pns & The most posterior point of the hard palate \\
\hline pt & Posterior tongue \\
\hline ppw & Posterior pharyngeal wall \\
\hline $\mathbf{S}$ & Mid-point of sella turcica \\
\hline stern & Sternum \\
\hline $\mathbf{u}$ & Uvula \\
\hline ut & Uvula tip \\
\hline
\end{tabular}




\section{Table 2 Variables chosen to describe vocal tract-related changes in subjects asked to hum low and high notes}

\section{Craniocervical}

$\mathrm{cvt} / \mathrm{nsl} \quad$ The angle between $\mathrm{cvt}$ and nsl

evt/nsl The angle between evt and nsl

opt/nsl The angle between opt and nsl

Angular

ae

The angle of the epiglottis (intersection of ans-pns with line joining 2 points on epiglottis)

asp

The angle between lines joining ans - pns and pns-ut

\section{Craniocaudal}

et-nsl

hy-nsl

1-hy

1-nsl

pns-ut

stern-hy

ut-nsl
The perpendicular distance of epiglottis tip from nasion-sella line

The perpendicular distance of hyoid bone from nasion-sella line

The anterior point of vocal folds to the antero-superior margin of hyoid bone

The perpendicular distance of larynx from nasion-sella line

The distance between posterior nasal spine and uvula tip

The distance from sternum to antero-superior point of hyoid body

The perpendicular distance of uvula tip from nasion-sella line

\section{Antero-posterior}

\begin{tabular}{ll}
\hline c3-me & The antero-inferior point of C3 to menton \\
et-ppw & The epiglottis tip to posterior pharyngeal wall \\
hy-c3 & The antero-superior point of hyoid body to antero-inferior point of C3 \\
hy-ma & The antero-superior point of hyoid body to posterior cortex of mandible \\
hy-me & The antero-superior point of hyoid body to menton \\
ltw & The superior margin of arytenoid cartilage to base of epiglottis, perpendicular to airway \\
pt-ppw-et & The posterior tongue to posterior pharyngeal wall at epiglottis tip \\
pt-ppw-ut & The posterior tongue to posterior pharyngeal wall at uvula tip \\
u-ppw & The minimal distance between uvula and posterior pharyngeal wall \\
ut-ppw & The distance between the tip of the uvula and the posterior pharyngeal wall
\end{tabular}


Table 3 One way repeated measures ANOVA for Rest, LNH and $\mathrm{HNH}^{1}$

\begin{tabular}{|c|c|c|c|c|c|c|c|}
\hline \multirow{3}{*}{ Variables } & \multirow{3}{*}{$\begin{array}{c}\text { Rest } \\
\text { Mean (SD) Range }\end{array}$} & \multirow{3}{*}{$\begin{array}{c}\text { LNH } \\
\text { Mean (SD) Range }\end{array}$} & \multirow[b]{2}{*}{ Mean (SD) Range } & \multirow{2}{*}{$P$ Value } & \multicolumn{3}{|c|}{ Post-hoc Analysis } \\
\hline & & & & & \multicolumn{3}{|c|}{ (critical levels $0.017,0.025,0.050$ ) } \\
\hline & & & & & Rest-LNH & Rest-HNH & $\overline{\mathrm{LNH}-\mathrm{HNH}}$ \\
\hline opt/nsl & $\mathbf{1 0 2 . 2}(7.0) 93-115.6$ & $\mathbf{1 0 1 . 4}(6.7) 88.3-110.4$ & $\mathbf{1 0 5 . 4}(8.2) 95.4-119.5$ & 0.008 & 0.515 & 0.015 & 0.003 \\
\hline $\mathrm{cvt} / \mathrm{nsl}$ & $\mathbf{1 0 1 . 9}(7.0) 92.8-115$ & $101.1(6.8) 90.6-108.1$ & $\mathbf{1 0 5 . 4}(7.5) 95.4-118.4$ & 0.002 & 0.496 & 0.004 & $<0.001$ \\
\hline evt/nsl & $103.3(7.7) 90.9-118.6$ & $\mathbf{1 0 7 . 4}(9.0) 91.5-120.5$ & $\mathbf{1 0 7 . 1}(7.1) 92.7-119.1$ & 0.018 & 0.011 & 0.016 & 0.851 \\
\hline ae & $85.2(7.6) 71.8-95.4$ & 93.8 (13.6) 68-109.8 & 85.2 (14.7) 59.4-110.2 & 0.115 & & & \\
\hline asp & $\mathbf{1 3 2 . 5}(5.8) 121.8-139.3$ & $133.0(8.2) 118.3-145$ & $134.2(6.6) 124.6-142.8$ & 0.756 & & & \\
\hline pns-ut & $\mathbf{3 9 . 3}(5.7) 30.6-50.0$ & 39.9 (3.7) $34.7-46.0$ & $37.2(4.7) 31.8-47.4$ & 0.08 & & & \\
\hline $\mathrm{u}-\mathrm{ppw}$ & $4.1(2.4) 0.0-7.0$ & $4.8(2.4) 6.6-13.6$ & 4.3 (3.1) $2.7-14.5$ & 0.783 & & & \\
\hline ut-ppw & $\mathbf{8 . 6}(2.5) 4.4-11.9$ & $9.4(2.4) 6.6-13.6$ & 7.9 (3.0) $2.7-14.5$ & 0.13 & & & \\
\hline ut-nsl & $71.5(8.8) 59.8-84.8$ & $71.4(8.7) 54.0-79.5$ & $\mathbf{6 8 . 6}(8.7) 54.9-78.1$ & 0.088 & & & \\
\hline et-nsl & $87.9(9.1) 76.3-101.0$ & $\mathbf{9 0 . 8}(7.5) 76.8-99.9$ & 87.5 (11.0) $77.2-108.3$ & 0.13 & & & \\
\hline hy-nsl & $\mathbf{1 0 6 . 4}(11.5) 89.7-125.1$ & $113.4(9.1) 98.5-126.9$ & $\mathbf{1 0 8 . 5}(11.9) 90.1-133$ & $<0.001$ & $<0.001$ & 0.152 & 0.003 \\
\hline 1-nsl & $\mathbf{1 2 9 . 0}(15.1) 111.2-152$ & 132.7 (13.5) $114.1-150.8$ & $\mathbf{1 2 5 . 5}(19.7) 102.9-163.1$ & 0.012 & 0.1 & 0.121 & 0.003 \\
\hline 1-hy & $\mathbf{2 4 . 2}(5.0) 15.7-31.0$ & $22.1(5.1) 13.6-28.1$ & $21.4(7.5) 12.6-35.2$ & 0.11 & & & \\
\hline hy-c3 & 36.7 (3.7) $30.6-41.2$ & 39.9 (7.7) $29.5-55.1$ & $44.1(6.4) 32.8-56.0$ & 0.003 & 0.098 & $<0.001$ & 0.031 \\
\hline c3-me & $\mathbf{8 2 . 8}(7.8) 75.7-94.7$ & $82.5(7.7) 71.2-95.1$ & $86.6(8.0) 75.4-99.4$ & 0.003 & 0.797 & 0.004 & 0.002 \\
\hline hy-me & $47.2(6.4) 38.3-57.6$ & 45.4 (7.6) 38.0-56.1 & 43.8 (7.3) 33.0-56.1 & 0.152 & & & \\
\hline hy-ma & 37.5 (5.9) 28.7-49.2 & $\mathbf{3 8 . 0}(7.6) 28.3-51.8$ & $33.6(7.0) 25.3-46.4$ & 0.038 & 0.811 & 0.033 & 0.02 \\
\hline ltw & $\mathbf{9 . 0}(2.6) 4.9-12.5$ & $6.2(3.4) 3.4-15.0$ & $8.4(3.9) 3.2-14.5$ & 0.075 & & & \\
\hline stern-hy & $\mathbf{1 1 0 . 1}(15.2) 81.4-135.6$ & $\mathbf{1 0 0 . 1}(16.3) 81.2-136.6$ & $\mathbf{1 0 8 . 7}(18.1) 85.0-144.8$ & $<0.001$ & 0.001 & 0.405 & 0.009 \\
\hline pt-ppw-ut & $\mathbf{8 . 6}(2.5)$ 5.72-17.2 & $9.4(2.4) 8.8-28.6$ & $7.9(3.0) 4.8-33.0$ & 0.148 & & & \\
\hline pt-ppw-et & $13.2(2.8) 9.7-17.2$ & $\mathbf{1 7 . 6}(5.2) 10.6-24.2$ & 15.4 (7.1) 7.5-28.6 & 0.098 & & & \\
\hline et-ppw & 8.1 (1.9) 4.4-10.1 & $\mathbf{1 2 . 8}(5.6) 4.4-22.4$ & $11.2(5.5) 3.5-22.0$ & 0.041 & 0.014 & 0.09 & 0.365 \\
\hline
\end{tabular}

\footnotetext{
${ }^{1}$ Angular measures are in degrees and all linear measurements are in $\mathrm{mm}$.
} 
Figure 2 Significant differences between $\mathrm{LNH}$ and $\mathrm{HNH}$, Rest and $\mathrm{LNH}$, and Rest and $\mathrm{HNH}$ (solid line indicates increasing dimensions, dashed line indicates decreasing dimensions)

$2.1 \mathrm{LNH} \neq \mathrm{HNH}$

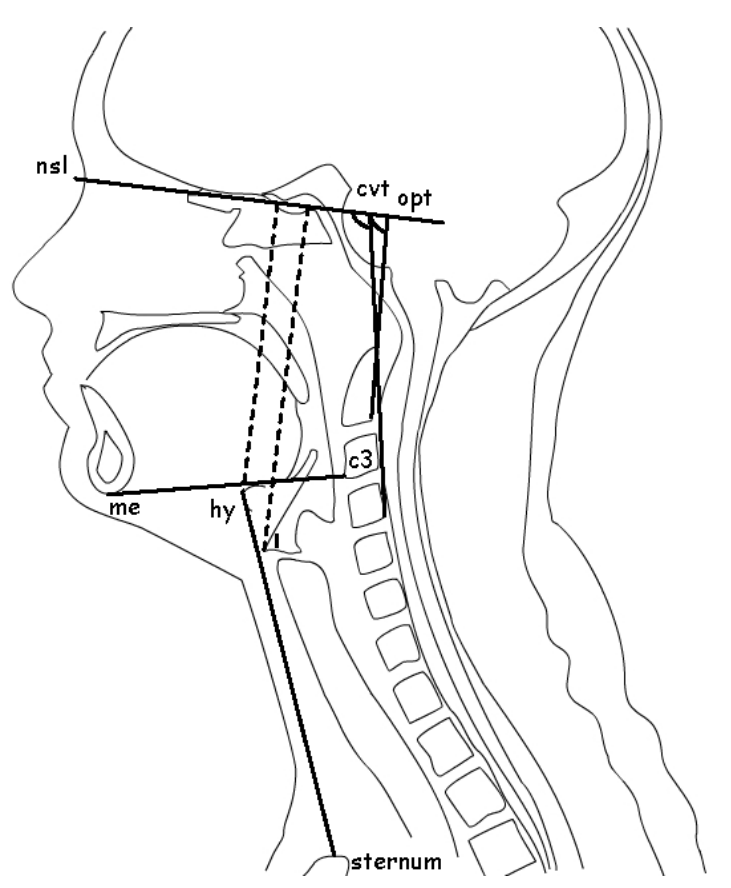

2.2 Rest $\neq \mathrm{LNH}$

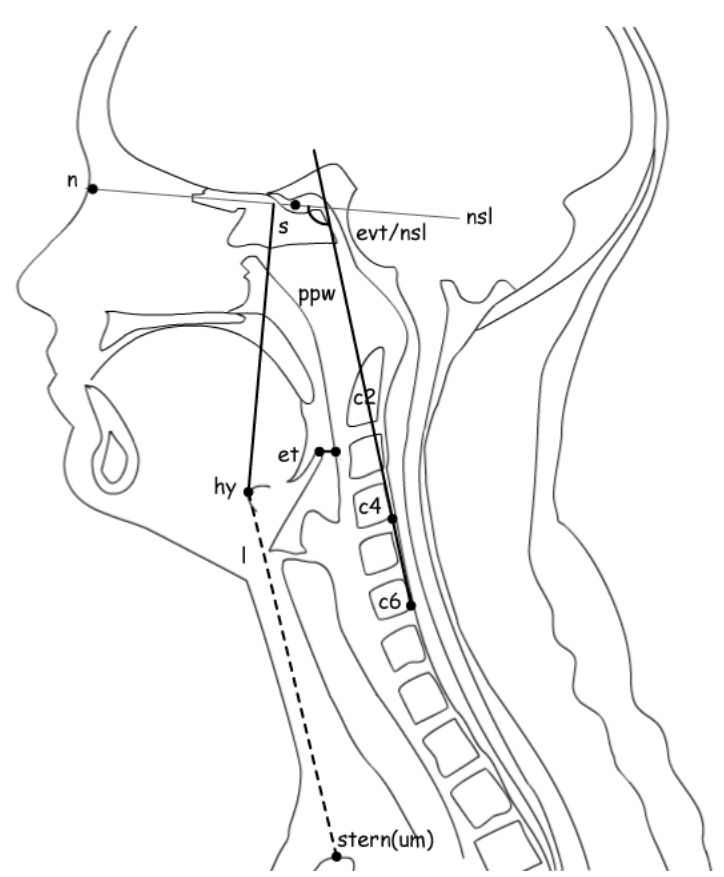

$2.3 \quad$ Rest $\neq \mathrm{HNH}$

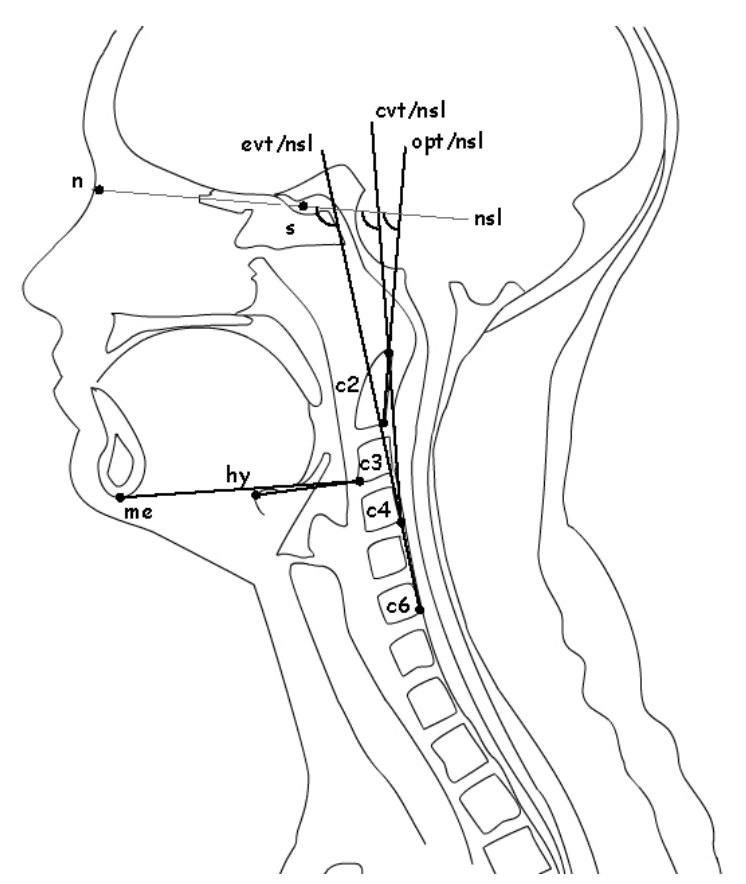


Table 4 Correlations observed for $\mathrm{LNH}^{2}$

\begin{tabular}{|c|c|c|c|c|c|c|c|c|c|c|c|c|c|c|c|c|c|c|c|}
\hline Variable & $t / n$ & /nsl & hy-nsl & I-nsl & et-nsl & ut-nsl & asp & pns-ut & stern-hy & Itw & hy-c3 & 3 hy-me & hy-ma & ae & u-ppw & c3-me & pt-ppv & ut pt-ppw & t et-ppw \\
\hline$t / n s l$ & E & $0.97 *$ & $-0.67 *$ & -0.57 & -0.62 & -0.61 & $0.75^{*}$ & -0.37 & 0.31 & 0.27 & 0.37 & -0.06 & -0.11 & -0.06 & 0.08 & $0.66^{*}$ & -0.31 & 0.07 & 0.41 \\
\hline$t / n s l$ & $0.97 * * *$ & - & $-0.72 *$ & $-0.71^{*}$ & $-0.73^{*}$ & $-0.68^{*}$ & $0.74 *$ & -0.53 & 0.37 & 0.42 & 0.49 & -0.22 & -0.3 & -0.12 & 0.18 & 0.59 & -0.32 & 0.05 & 0.46 \\
\hline$y-n s l$ & $-0.67 *$ & $-0.72 *$ & - & $0.71^{*}$ & $0.74 *$ & 0.54 & -0.46 & 0.51 & $-0.67 *$ & -0.61 & -0.39 & 0.44 & 0.42 & 0.29 & -0.06 & -0.32 & 0.26 & -0.13 & -0.53 \\
\hline -nsl & -0.57 & $-0.64 *$ & $0.71^{*}$ & - & $0.9 * * *$ & 0.56 & -0.4 & $0.71^{*}$ & -0.47 & -0.5 & -0.42 & 0.33 & 0.42 & 0.33 & -0.31 & -0.19 & 0.02 & -0.21 & -0.53 \\
\hline$-\mathbf{n s l}$ & -0.62 & $-0.73^{*}$ & $0.76^{*}$ & $0.9 * * *$ & - & $0.8^{* * *}$ & $-0.66^{*}$ & $0.76^{*}$ & -0.51 & -0.38 & -0.24 & 0.24 & 0.31 & 0.08 & 0.01 & -0.21 & -0.05 & -0.42 & $-0.7^{*}$ \\
\hline -nsl & -0.61 & $-0.68^{*}$ & 0.54 & 0.56 & $0.8 * *$ & - & $-0.8^{*}$ & $0.8 * *$ & -0.46 & -0.39 & -0.32 & 0.33 & 0.34 & -0.18 & 0.16 & -0.31 & -0.15 & -0.57 & -0.84 \\
\hline spp & $0.75^{*}$ & $0.74 *$ & -0.46 & -0.4 & $-0.66 *$ & $-0.8 * *$ & - & -0.37 & 0.21 & 0.06 & 0.17 & 0.02 & -0.08 & 0.29 & -0.22 & 0.59 & -0.07 & 0.39 & 0.6 \\
\hline ns-ut & -0.37 & -0.53 & 0.51 & $0.71^{*}$ & $0.76 *$ & $0.8^{* * *}$ & -0.37 & - & $-0.69 *$ & $-0.69 *$ & -0.42 & 0.61 & 0.53 & 0.24 & 0.03 & 0.12 & -0.14 & -0.32 & -0.65 \\
\hline rn-hy & 0.31 & 0.37 & $-0.67 *$ & -0.47 & -0.51 & -0.46 & 0.21 & $-0.69 *$ & - & $0.69 *$ & 0.24 & -0.52 & -0.29 & -0.41 & -0.37 & -0.18 & 0.12 & 0.2 & 0.43 \\
\hline $\boldsymbol{w}$ & 0 & 0. & -0 & -0.5 & -0.38 & -0.39 & 0.06 & * & 0. & 5 & 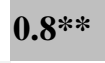 & 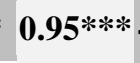 & * & $-0.65^{*}$ & 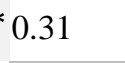 & 0.06 & -0 & -0.12 & 0.41 \\
\hline$y-c 3$ & 0.37 & 0.49 & -0.39 & -0.42 & -0.24 & -0.32 & 0.17 & -0.42 & 0.24 & $0.8^{* *}$ & - & $-0.8^{* *}$ & $-0.9 * *$ & -0.59 & $0.69 *$ & 0.53 & -0.36 & -0.1 & 0.42 \\
\hline -me & -0.06 & -0.22 & 0.44 & 0.33 & 0.24 & 0.33 & 0.02 & 0.61 & -0.52 & $0.95 * * *$ & $-0.8 * *$ & *_- & 0.93 & 0.61 & -0.34 & -0.02 & 0.31 & 0.12 & -0.39 \\
\hline . & -0.11 & -0.3 & 0.42 & 0.42 & 0.31 & 0.34 & -0.08 & 0.53 & -0.29 & $-0.86 * *$ & $-0.9^{* * *}$ & * 0.93 & - & 0.54 & -0.54 & -0.19 & 0.4 & 0.15 & -0.4 \\
\hline 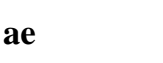 & -0.06 & -0.12 & 0.29 & 0.33 & 0.08 & -0.18 & 0.29 & 0.24 & -0.41 & $-0.65^{*}$ & -0.59 & 0.61 & 0. & - & -0.38 & -0.02 & 0. & 0. & -0.02 \\
\hline ppw & 0.08 & 0.18 & -0.06 & -0.31 & 0.01 & 0.16 & -0.22 & 0.03 & -0.36 & 0.31 & $0.69^{*}$ & -0.34 & -0.54 & -0.38 & - & 0.44 & -0.30 & -0.24 & 0.02 \\
\hline 3-me & $0.66^{*}$ & 0.59 & -0.32 & -0.19 & -0.21 & -0.31 & 0.59 & 0.11 & -0.18 & 0.06 & 0.53 & -0.02 & -0.19 & -0.02 & 0.44 & - & -0.24 & 0.19 & 0.02 \\
\hline$t-p p$ & -0.31 & -0.32 & 0.26 & 0.02 & -0.05 & -0.15 & -0.07 & -0.14 & 0.12 & -0.29 & -0.36 & 0.31 & 0.42 & 0.4 & -0.3 & -0.24 & - & $0.8 * *$ & 0.33 \\
\hline -ppw-e & 7 & 0.05 & -0.13 & -0.21 & $0.78^{* * *}$ & -0.57 & 0.39 & -0.32 & 0.2 & -0.12 & -0.1 & 0.12 & 0.15 & 0.38 & -0.24 & 0.19 & $0.8 * *$ & - & $0.78^{* *}$ \\
\hline -ppw & 0.41 & 0.46 & -0.53 & -0.53 & $-0.7 *$ & $-0.84 *$ & 0.6 & $-0.65^{*}$ & 0.43 & 0.41 & 0.42 & -0.4 & -0.4 & -0.02 & 0.02 & 0.43 & 0.33 & $0.78 * *$ & - \\
\hline
\end{tabular}

$P<0.05(*), P<0.01(* *), P<0.001(* * *)$ 
Table 5 Correlations observed for HNH $\mathrm{P}<0.05(*), \mathrm{P}<0.001(* *), \mathrm{P}<0.001(* * *)$

\begin{tabular}{|c|c|c|c|c|c|c|c|c|c|c|c|c|c|c|c|c|c|c|}
\hline \multirow{3}{*}{$\begin{array}{l}\text { Variable } \\
\text { opt/nsl } \\
\text { cvt/nsl }\end{array}$} & \multicolumn{2}{|c|}{ opt/nsl cvt/nsl } & \multirow{2}{*}{$\frac{\text { l-hy }}{0}$} & \multirow{2}{*}{$\frac{\text { l-nsl }}{-0.23}$} & \multirow{2}{*}{$\frac{\text { et-nsl }}{-0.27}$} & \multirow{2}{*}{$\frac{\text { ut-nsl }}{-0.56}$} & \multirow{2}{*}{$\frac{\text { asp }}{0.62}$} & \multirow{2}{*}{$\frac{\text { ltw }}{-0.32}$} & \multicolumn{3}{|c|}{ ut-ppw u-ppw stern-hy } & \multirow{2}{*}{$\frac{\text { y hy-c3 }}{0.4}$} & \multirow{2}{*}{$\frac{\text { hy-me }}{-0.05}$} & \multirow{2}{*}{$\frac{\text { hy-ma }}{-0.33}$} & \multicolumn{4}{|c|}{ c3-me pt-pw-ut pt-ppw-et et-ppw } \\
\hline & - & $0.82 * *$ & & & & & & & 0.03 & 0.37 & -0.1 & & & & $0.81 * *$ & 0.11 & 0.15 & 0.16 \\
\hline & $0.82 * *$ & - & 0.01 & -0.15 & -0.24 & $-0.73 *$ & 0.54 & -0.01 & 0.15 & 0.62 & 0.11 & 0.47 & -0.13 & -0.36 & 0.62 & 0 & 0 & 0.19 \\
\hline l-hy & 0 & 0.01 & - & $0.67 *$ & 0.49 & 0.2 & -0.48 & 0.49 & 0.17 & 0.09 & -0.14 & 0.36 & -0.37 & 0.37 & -0.23 & -0.45 & 0.17 & 0.07 \\
\hline l-nsl & -0.23 & -0.15 & $0.67 *$ & 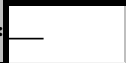 & $0.88 * * *$ & 0.35 & -0.55 & 0.44 & 0.1 & 0.19 & -0.31 & -0.32 & 0.43 & $0.64 *$ & -0.17 & -0.22 & 0.27 & $0.65 *$ \\
\hline et-nsl & -0.27 & -0.24 & 0.49 & $0.88 * * *$ & - & 0.41 & -0.51 & 0.13 & -0.17 & 0 & -0.37 & -0.36 & 0.52 & 0.56 & 0.16 & -0.15 & 0.16 & 0.36 \\
\hline ut-nsl & -0.56 & $-0.73 *$ & 0.2 & 0.35 & 0.41 & - & $-0.77 * *$ & 0.33 & 0.05 & -0.23 & -0.59 & $-0.7 *$ & 0.51 & 0.49 & -0.23 & 0.22 & 0.36 & 0.02 \\
\hline asp & 0.62 & 0.54 & 0.01 & -0.15 & -0.24 & $-0.77 * *$ & - & $-0.63 *$ & 0.15 & 0.62 & 0.11 & 0.47 & -0.13 & -0.36 & 0.62 & 0 & 0 & 0.19 \\
\hline Itw & -0.32 & -0.01 & 0.49 & 0.44 & 0.13 & 0.33 & $-0.63 *$ & - & $0.76^{*}$ & 0.53 & -0.11 & -0.2 & 0.02 & 0.19 & -0.42 & -0.13 & 0.18 & 0.44 \\
\hline ut-ppw & 0.03 & 0.15 & 0.17 & 0.1 & -0.17 & 0.05 & -0.21 & $0.76^{*}$ & 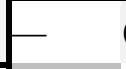 & $0.76^{*}$ & -0.31 & -0.06 & -0.02 & 0.01 & -0.01 & -0.36 & -0.08 & 0.39 \\
\hline u-ppw & 0.37 & 0.62 & 0.09 & 0.19 & 0 & -0.23 & 0.09 & 0.53 & $0.76^{*}$ & - & -0.21 & 0.13 & 0.05 & -0.02 & 0.36 & -0.23 & -0.05 & 0.39 \\
\hline stern-hy & -0.1 & 0.11 & -0.14 & -0.31 & -0.37 & -0.59 & 0.44 & -0.11 & -0.31 & -0.21 & - & 0.56 & $-0.74 *$ & -0.4 & -0.48 & -0.15 & -0.48 & -0.36 \\
\hline hy-c3 & 0.4 & 0.47 & -0.37 & -0.32 & -0.36 & $-0.7^{*}$ & 0.49 & -0.2 & -0.06 & 0.13 & 0.56 & - & $-0.86 * *$ & $-0.79 * *$ & 0.02 & 0.3 & 0.07 & 0.16 \\
\hline hy-me & -0.05 & -0.13 & 0.3 & 0.43 & 0.52 & 0.51 & -0.3 & 0.02 & -0.02 & 0.05 & $-0.74 *$ & $-0.86 * *$ & - & $0.8^{* * *}$ & 0.39 & -0.17 & 0.11 & 0.06 \\
\hline hy-ma & -0.33 & -0.36 & 0.37 & $0.64^{*}$ & 0.56 & 0.49 & -0.03 & -0.4 & 0.01 & -0.02 & -0.4 & $-0.79 * *$ & $0.8^{* * *}$ & - & -0.01 & -0.3 & 0.03 & 0.21 \\
\hline c3-me & $0.81 * *$ & 0.62 & -0.23 & -0.17 & -0.06 & -0.23 & 0.5 & -0.42 & -0.01 & 0.36 & -0.48 & 0.02 & 0.39 & -0.01 & - & 0.21 & 0.2 & 0.07 \\
\hline pt-ppw-ut & 0.11 & 0 & -0.45 & -0.22 & -0.15 & 0.22 & -0.07 & -0.13 & -0.36 & -0.23 & -0.15 & 0.3 & -0.17 & -0.3 & 0.21 & - & $0.78 * *$ & 0.14 \\
\hline pt-ppw-et & 0.15 & 0 & 0.07 & 0.27 & 0.16 & 0.36 & -0.39 & 0.18 & -0.08 & -0.05 & -0.48 & 0.07 & 0.11 & 0.03 & 0.2 & $0.78 * *$ & - & $0.65 *$ \\
\hline et-ppw & 0.16 & 0.19 & 0.36 & $0.65 *$ & 0.36 & 0.02 & -0.32 & 0.44 & 0.39 & 0.39 & -0.36 & 0.16 & 0.06 & 0.21 & 0.07 & 0.14 & $0.65 *$ & - \\
\hline
\end{tabular}


Table 6 Correlations common to LNH and HNH

$(+)$ indicates positive correlations (-) indicates negative correlations

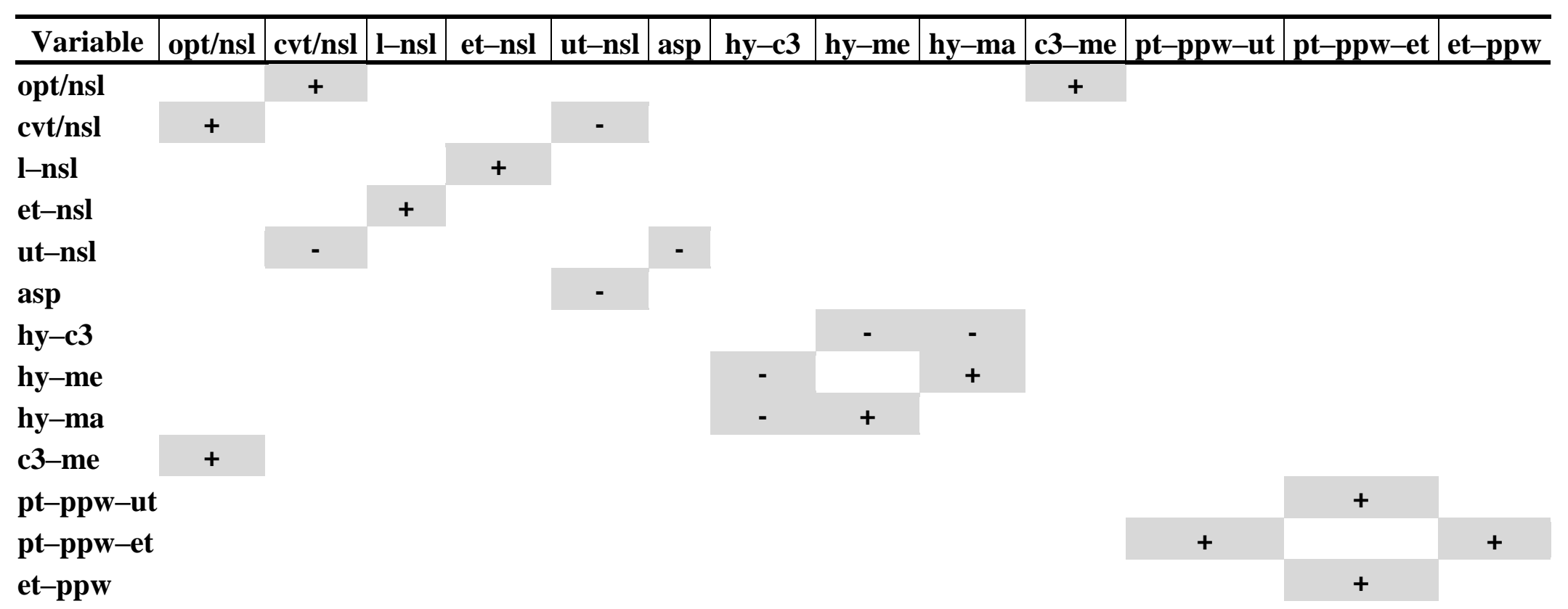


Figure 3 Correlations associated with hy-nsl, pns-ut and ltw in LNH, and hy-c3 in LNH and HNH
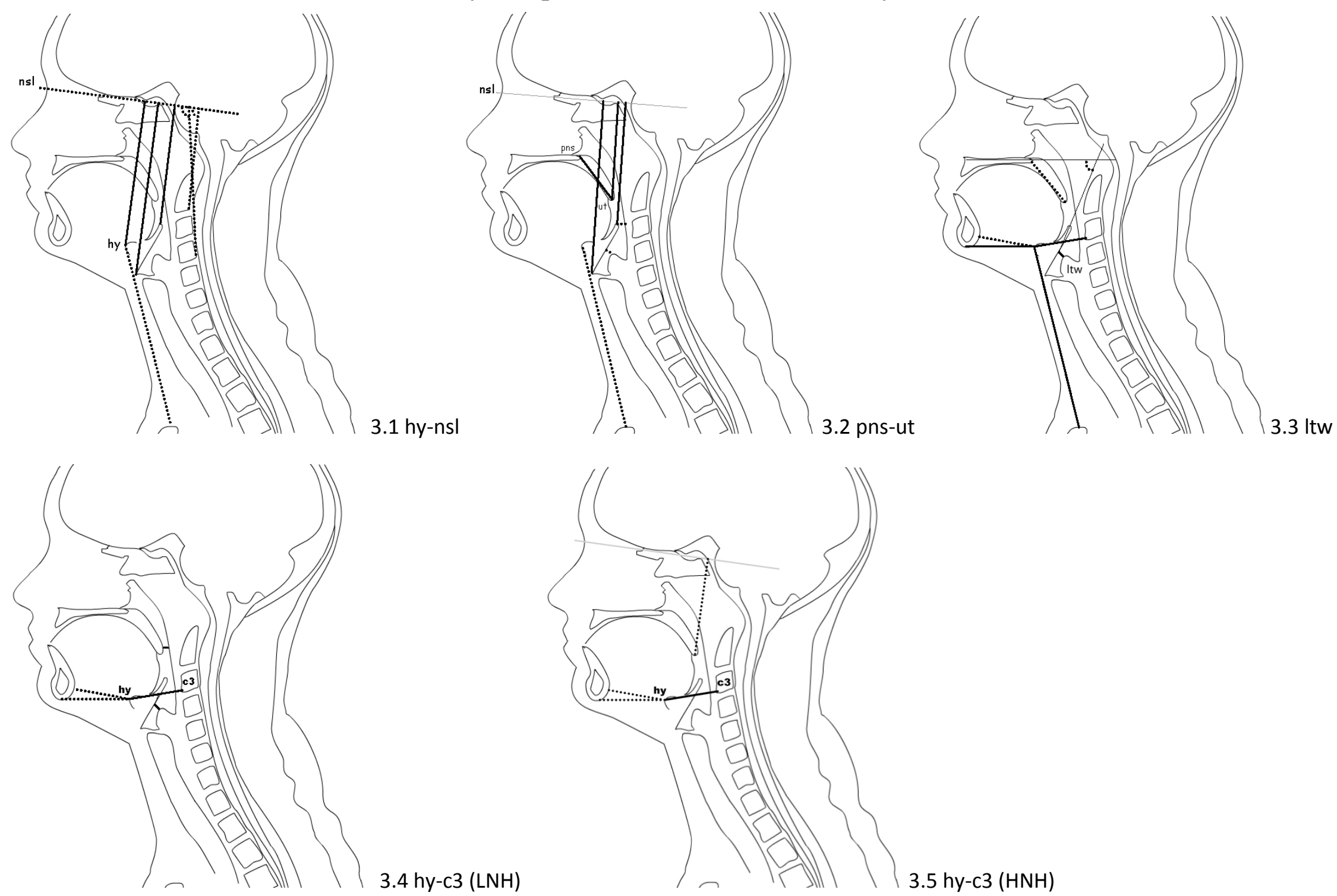
Figure 4 Superimposed tracings of images acquired from Volunteer 3 while humming low and high notes (the solid line represents LNH and dotted line represents HNH)

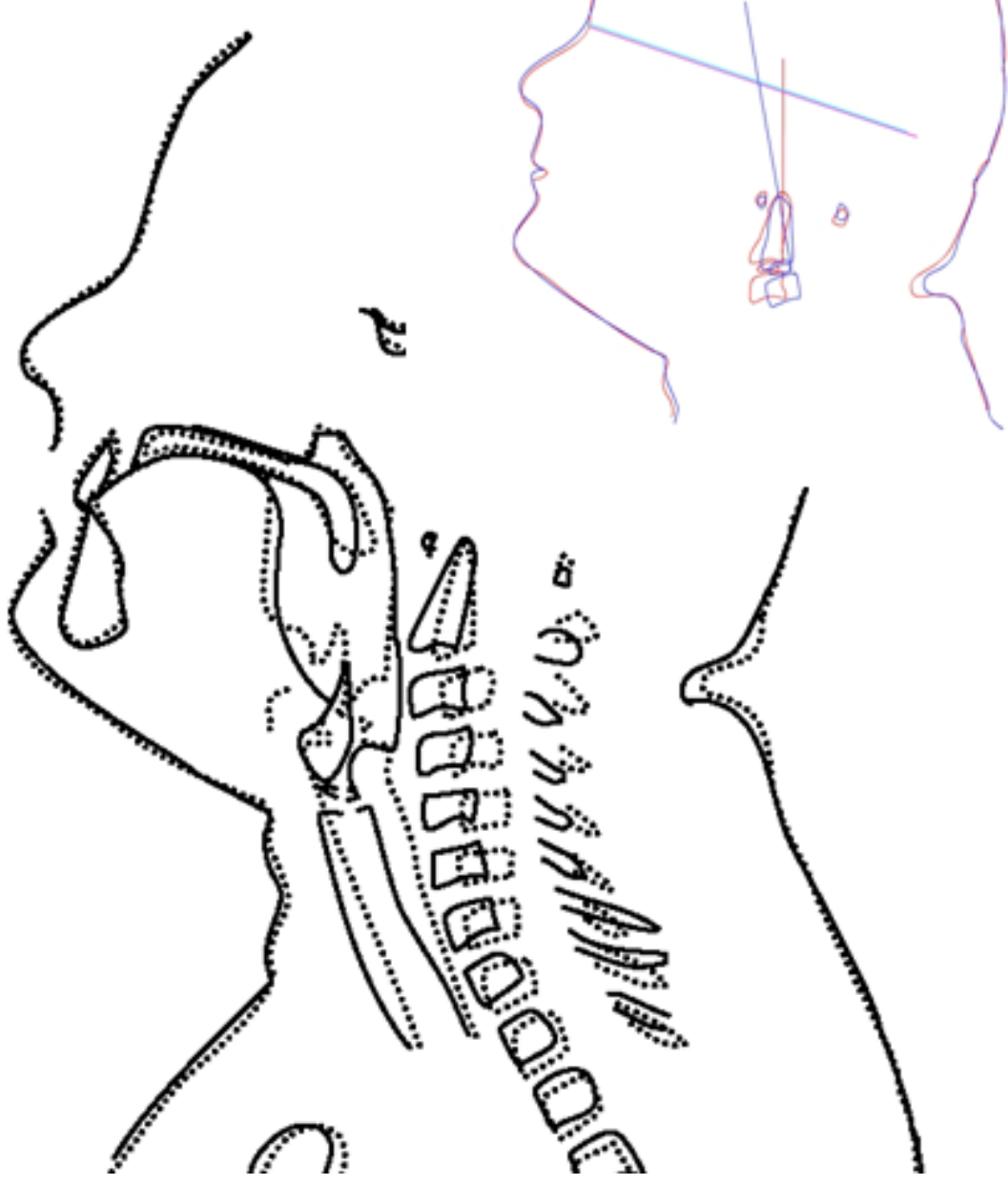

